B. Wulfhorst
H. J. Schwanitz

\section{Gesundheitspädagogik in der Prävention von Berufsdermatosen}

Health Education in the Prevention of Occupational Skin Diseases

\section{Zusammenfassung}

Die Implementierung gesundheitspädagogischer Maßnahmen in den Zusammenhang der Prävention berufsbedingter Erkrankungen gewinnt zunehmend an Bedeutung. In diesem Kontext werden die Möglichkeiten pädagogischer Interventionen dargestellt. Ziel dieses Beitrages ist es, die unterschiedliche Relevanz pädagogischer und berufsdermatologischer Ansätze in den drei Bereichen der Prävention (primär, sekundär, tertiär) zu verdeutlichen. Die Effektivität und auch Effizienz von Präventionsprogrammen, die auf einer Integration berufsdermatologischer und (gesundheits-)pädagogischer Interventionen basieren, ist in kontrollierten Studien eindeutig belegt worden. An Beispielprojekten („Interventionsstudie bei Auszubildenden des Friseurhandwerks“, „Sekundäre Individualprävention im Friseurhandwerk“, „Stationäre Präventionsmaßnahme für hautkranke Versicherte“) werden Ziele, Methoden, Inhalte und Ergebnisse dargestellt.

\section{Abstract}

In addition to intensified dermatological treatment, health education measures have proved to be effective in the framework of different projects carried out at the University of Osnabrück on primary, secondary and tertiary prevention in the case of patients with occupational skin disorders. They worked both in the case of affected persons in the sense of behaviour prevention as well as in the affected working environments in the sense of condition prevention. The conclusion of the projects results is that the prevention of occupationally skin diseases and maintenance of health may be achieved by influencing the individual behavior via educational programs in addition to medical care.
Die Bedeutung gesundheitspädagogischer Interventionen im Rahmen der Prävention und Rehabilitation von Hauterkrankungen und hier insbesondere von Berufsdermatosen wird erst seit wenigen Jahren verstärkt diskutiert. Auf die Notwendigkeit pädagogischer Interventionen als Ergänzung zu medizinischer Diagnostik, Beratung und Therapie haben jedoch vor mehr als 20 Jahren bereits Hornstein et al. [1] hingewiesen, die ausführen, dass „... weniger medizinische als psychosoziale Faktoren für die erfolgreiche Rehabilitation chronisch Hautkranker verantwortlich“ seien ... und dass insbesondere „... die praktische Anleitung und ,Erziehung، der Patienten zu konsequenter Nachbe- handlung und zur Vorbeugung arbeitsbedingter Hautschäden das schwierigste Problem“ darstelle. Im Folgenden soll unter besonderer Berücksichtigung eigener Forschungsergebnisse die Integration der Gesundheitspädagogik in die Prävention und Rehabilitation von berufsbedingten Hauterkrankungen dargestellt werden. Eine kurze Begriffsdefinition sei vorangestellt: Gesundheitspädagogik beschäftigt sich mit Erkenntnissen und Methoden zur (unmittelbaren und mittelbaren) Beeinflussung des gesundheitsfördernden persönlichen und sozialen Verhaltens von Einzelnen und Gruppen [2]. Zentraler Gegenstand dieser pädagogischen Teildisziplin ist demnach die Beeinflussung des ge- 
sundheitsrelevanten Verhaltens. Auf den Ebenen von Wissen, Einstellungen, Fertigkeiten und Kompetenzen, zu deren Erwerb und Umsetzung immer auch die Motivation der Patienten eine große Rolle spielt, soll Einfluss genommen werden auf den Informationsstand der Betroffenen, auf Gewohnheiten, Verdrängungsstrategien, tradierte Verhaltensmuster etc. [3]. Bezüglich der Handlungskompetenz geht es u. a. auch darum, konkrete Verhaltensweisen einzuüben, zu trainieren [4].

Schwerpunkt der im Folgenden dargestellten kontrollierten Studien zur primären, sekundären und tertiären Prävention berufsbedingter Hauterkrankungen unter besonderer Berücksichtigung der gesundheitspädagogischen Aspekte sind die Hauterkrankungen im Friseurhandwerk. Hier treten überdurchschnittlich häufig berufsbedingte Hauterkrankungen auf und aufgrund des dramatischen Anstiegs der Meldungen einer Berufskrankheit Anfang der 90er-Jahre sind hier zahlreiche Forschungsprojekte durchgeführt worden $[5,6]$.

\section{Epidemiologische Basis}

Durch die im folgenden skizzierten Studien wird die epidemiologische Basis dargestellt, durch die u.a. gesundheitspädagogische Interventionsstudien legitimiert werden. Uter et al. $[7,8]$ haben in einer großen ausbildungsbegleitenden, prospektiven Kohortenstudie $(n=2275)$ zeigen können, dass über ein Drittel der Friseurauszubildenden im Laufe ihrer dreijährigen Ausbildungszeit Hautveränderungen entwickelt haben, die überwiegend in den Interdigitalräumen der Finger lokalisiert waren. Diese Interdigitalraumekzeme waren nicht überdurchschnittlich häufig auf konstitutionell empfindlicher (atopischer) Haut anzutreffen, was den starken schädigenden Effekt der Feuchtarbeit und indirekt den potenziellen Nutzen eines verbesserten Hautschutzes am Arbeitsplatz verdeutlicht. Über eine Langzeitstudie mit direktem gesundheitspädagogischen Hintergrund berichten Schlesinger et al. [9]. Durch kontinuierliche schriftliche Befragung von Auszubildenden des Friseurhandwerks in Niedersachsen in 5-Jahres-Abständen $1989(\mathrm{n}=$ 8256), $1994(\mathrm{n}=4967)$ und 1999 $(n=3741)$, der Rücklauf betrug zwischen 49 und 65\% (Tab.1), sollte der jeweilige Status-quo von Hauterkrankungen und Hautschutz bei Auszubildenden in diesem Beruf erhoben werden. Es handelte sich jeweils um Totalerhebungen, wobei die subjektiven Angaben der Friseurauszubildenden erhoben wurden.

Tab. 1 Stichprobengröße und Rücklaufquote der Totalerhebungen bei Friseurauszubildenden in Niedersachsen 1989, 1994 and 1999

\begin{tabular}{|llll|}
\hline & $\begin{array}{l}\text { Auszubildende } \\
\text { gesamt }(\boldsymbol{n})\end{array}$ & Rücklauf $(\boldsymbol{n})$ & Rücklauf in \% \\
\hline 1986 & 8256 & 4008 & 48,5 \\
\hline 1994 & 4967 & 2505 & 50,8 \\
\hline 1999 & 3741 & 2427 & 64,9 \\
\hline
\end{tabular}

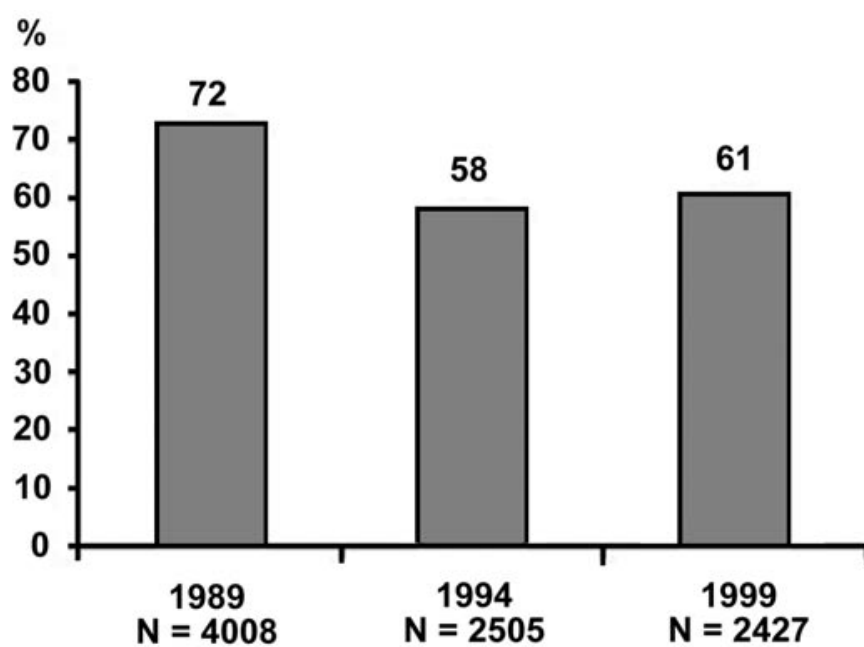

Abb. 1 Totalerhebung bei Friseurauszubildenden in Niedersachsen, Hautveränderungen während der Ausbildung.

Die Inzidenz berufsbedingter Hautveränderungen bei Friseurauszubildenden ist von 1989 auf 1994 im Untersuchungskollektiv deutlich gesunken, von 1994 auf 1999 ist wiederum ein leichter Anstieg zu verzeichnen (Abb.1).

Als Begründung für diesen Anstieg können weitere Ergebnisse dieser Studie herangezogen werden: Während 1989 rund 16\% der Befragten, die angaben, unter Hautveränderungen zu leiden, einen Hautarzt aufgesucht haben, waren dies 1994 48\% und 1999 nur 29\%. Das eigene Hautschutzverhalten schätzten 1999 55,7\% als „verbesserungswürdig“ ein, 39\% als „hinreichend“ und $22 \%$ stuften dieses als „sehr gut" ein. Bei Auszubildenden mit angegebenem „hinreichenden“ Hautschutzverhalten war allerdings festzustellen, dass etwa die Hälfte bei Tätigkeiten wie Haarewaschen, Auftragen von Dauerwellmitteln und beim Fixieren von Dauerwellen entgegen der in der einschlägigen Arbeitsschutzvorschrift gemachten Vorgaben keine Handschuhe verwendeten [10].

\section{Primäre Prävention}

In direkter Konsequenz aus den Ergebnissen der oben dargestellten epidemiologischen Studien ist von Budde-Wamhoff zunächst eine 6-stündige Unterrichtseinheit zum Thema Hauterkrankungen und Hautschutz entwickelt worden, die von Lehrern an berufsbildenden Schulen über einen Schulbuchverlag bezogen werden kann [11]. Zwischen 1996 und 1999 ist eine Interventionsstudie durchgeführt worden mit dem Ziel, die Entstehung von Hauterkrankungen bei Auszubildenden des Friseurhandwerks zu vermeiden. Dieser Studie lag ein interdisziplinäres Präventionskonzept zugrunde, das neben berufsdermatologischen besonders auf gesundheitspädagogischen Interventionen beruhte. Vorrangige Zielgröße dieser Studie war die Verhaltensprävention, Aspekte der Verhältnisprävention wurden durch die Ausstattung der Auszubildenden mit adäquaten Hautschutzmitteln und Schutzhandschuhen sowie durch die Durchführung von Betriebsberatungen ebenfalls berücksichtigt [12]. 


\section{Konzeption und Methoden}

Die Interventions- und die Kontrollgruppe bestand aus Auszubildenden des Friseurhandwerks, die ihre Ausbildung 1996 begonnen hatten. Die Interventionsgruppe $(n=73)$ wurde aus Schülern einer Berufsschule in Osnabrück, die Kontrollgruppe $(n=112)$ aus Schülern einer Berufsschule in Hannover rekrutiert. Beide Gruppen wurden über die gesamte Ausbildungsdauer zwischen August 1996 und Juni 1999 viermal dermatologisch untersucht, der Hautzustand wurde anhand eines standardisierten Scores erfasst. In der Interventionsgruppe wurden von einer Gesundheitspädagogin sechs Unterrichtssequenzen mit insgesamt 15 Unterrichtsstunden zum Thema Hauterkrankungen und Hautschutz im Friseurhandwerk durchgeführt. Der Wissensstand und die Einstellung der Schüler zu diesem Thema wurde in beiden Gruppen zu Beginn des ersten und zum Abschluss des zweiten Ausbildungsjahres mittels schriftlicher Befragung erhoben. Im Sinne der Berücksichtigung verhältnispräventiver Aspekte ist die Interventionsgruppe mit adäquaten Hautschutzmitteln und Schutzhandschuhen ausgestattet und es sind Betriebsberatungen in den Ausbildungssalons durchgeführt worden. Die Unterschiede bezüglich bestehender Hautveränderungen wurden mittels des Wilcoxon-Signed-Rank-Test für gruppierte Daten auf Signifikanz geprüft $(\alpha=0,05)$. Die Ergebnisse aus den Schülerbefragungen zu Einstellungen, Wissen und Verhalten wurden anhand des U-Tests nach Wilcoxon, Mann und Whitney auf statistische Signifikanz geprüft.

\section{Ergebnisse}

Die Erstuntersuchung der Studienkollektive hat ergeben, dass diese bezüglich konstitutioneller Faktoren vergleichbar sind, die Unterschiede zwischen den Kollektiven waren zu diesem Zeitpunkt statistisch nicht signifikant. Bezüglich der Inzidenz von berufsbedingten Hauterkrankungen konnte für das Jahr 1997 kein Unterschied zwischen den Gruppen festgestellt werden. Im Jahr 1998 waren 90\% der Interventionsgruppe im Vergleich zu $75 \%$ der Kontrollgruppe hauterscheinungsfrei, diese Unterschiede waren signifikant $(p<0,05)$. Zum Ende der Interventionsstudie waren $81 \%$ der Interventionsgruppe im Vergleich zu $66 \%$ der Kontrollgruppe hauterscheinungsfrei (Tab. 2).

Die Motivation zu konsequentem Hautschutz sowie das diesbezügliche Wissen war im ersten Ausbildungsjahr in der Interventionsgruppe signifikant größer. Bereits vier Wochen nach Interventionsbeginn zeigten die Teilnehmer der Interventionsgruppe ein deutlich konsequenteres Schutzverhalten, beispielsweise bei der Verwendung von Schutzhandschuhen. Im Verlauf der Studie konnte das adäquate Schutzverhalten in der Interventionsgruppe weiter optimiert werden, während in der Kontrollgruppe keine bzw. lediglich geringfügige Unterschiede zum Ausgangsverhalten festzustellen waren.

Hervorzuheben ist, dass mit der vorliegenden Interventionsstudie gezeigt werden konnte, dass ein Ausbildungsabbruch aufgrund von Hauterkrankungen in der Interventionsgruppe vermieden werden konnte. In der Kontrollgruppe haben 10\% der Teilnehmer ihre Ausbildung aufgrund von Hautproblemen abgebrochen.

\section{Sekundäre Prävention}

Im Rahmen der sekundären Prävention bei hauterkrankten Friseuren, die der zuständigen Berufsgenossenschaft (BGW, Berufsgenossenschaft für Gesundheitsdienst und Wohlfahrtspflege) durch einen Hautarztbericht oder eine BK-Anzeige gemeldet waren, ist ein Konzept entwickelt und erprobt worden, das den Erkrankten ein Verbleiben im Beruf ermöglichen sollte. Hierbei haben sich gesundheitspädagogische Maßnahmen neben einer intensivierten berufsdermatologischen Behandlung als sehr effektiv erwiesen. Aufgrund der Kombination von ärztlicher Betreuung, Einzelberatung, Kleingruppenseminaren und Betriebsberatungen sind Aspekte der Verhaltensprävention, die bei den erkrankten Personen ansetzt, und der Verhältnisprävention, die bei den betroffenen Betrieben ansetzt, gleichermaßen berücksichtigt worden [3].

\section{Konzeption und Methoden}

Zu Beginn des Projektes, in das 215 erkrankte Friseure integriert worden sind, erfolgte neben der berufsdermatologischen Diagnostik und der Festlegung eines Therapiekonzeptes eine Erhebung von vorhandenen Krankheits- bzw. Gesundheitskonzepten sowie zur Motivation, im Beruf zu verbleiben. Ein aufeinander aufbauendes Maßnahmenpaket beinhaltete dann ein fachtheoretisches und fachpraktisch ausgerichtetes Hautschutzseminar, in dem inhaltlich auf die Ursachen und Formen der beruflich bedingten Hauterkrankungen eingegangen wurde, die einzusetzenden Hautschutzmaßnahmen erarbeitet wurden und in einem praktischen Teil das Gelernte direkt umgesetzt werden konnte. Ein weiterer Bestandteil des Projektes waren Betriebsberatungen in den Salons der erkrankten Friseure, an denen alle Mitarbeiter und der Betriebsinhaber teilgenommen haben. Hier wurde ebenfalls auf die Entstehung der beruflich bedingten Hauterkrankun-

Tab. 2 Ergebnisse der kontrollierten Interventionsstudie bei Friseurauszubildenden

\begin{tabular}{|c|c|c|c|c|c|c|c|c|c|c|c|c|c|c|c|c|c|}
\hline & & \multicolumn{4}{|c|}{1996} & \multicolumn{4}{|c|}{1997} & \multicolumn{4}{|c|}{1998} & \multicolumn{4}{|c|}{1999} \\
\hline & & $I$ & $\%$ & $K$ & $\%$ & $I$ & $\%$ & $K$ & $\%$ & $I$ & $\%$ & $\kappa$ & $\%$ & $I$ & $\%$ & $\kappa$ & $\%$ \\
\hline (n) & & 73 & & 112 & & 50 & & 73 & & 39 & & 48 & & 41 & & 56 & \\
\hline \multirow[t]{2}{*}{ Schwere } & 1 & 10 & 13,7 & 26 & 23,3 & 19 & 38 & 22 & 30,1 & 4 & 10 & 8 & 16,7 & 7 & 19,5 & 11 & 19,6 \\
\hline & 2 & 0 & & 2 & 1,8 & 2 & 4 & 5 & 6,8 & 0 & & 3 & 2,1 & 0 & & 7 & 12,5 \\
\hline
\end{tabular}

I = Interventionsgruppe; $\mathrm{K}$ = Kontrollgruppe; 0 = keine, 1 = geringgradige, 2 = mittelgradige, 3 = schwere Hautveränderungen 
Tab. 3 Bausteine des Projekts „Sekundäre Individualprävention in Friseurbetrieben“

\begin{tabular}{ll}
\hline Projektmaßnahmen \\
\hline Phase 1: & $\begin{array}{l}\text { dermatologische Untersuchung, erstes Beratungsgespräch, } \\
\text { exploratives Interview }\end{array}$ \\
\hline Phase 2: & $\begin{array}{l}\text { Hautschutzseminar „Theorie und Praxis von Hauterkrankungen } \\
\text { und Hautschutz im Friseurhandwerk“ }\end{array}$ \\
Phase 3: & Betriebsberatung in den Salons der Teilnehmer \\
\hline Phase 4: & Abschlussseminar/Abschlussgespräch \\
\hline \hline
\end{tabular}

gen und Möglichkeiten ihrer Prävention durch Anwendung adäquater Hautschutzmittel eingegangen. Zum Abschluss der Maßnahmen ist für die Teilnehmer ein Rekapitulationsseminar durchgeführt worden. Hier sind Schwierigkeiten bei der Umsetzung der erlernten Schutzmaßnahmen diskutiert und gemeinsam Lösungsstrategien erarbeitet worden (Tab.3). Die Maßnahmen sind durch Befragungen mit unterschiedlichen Befragungsinstrumenten (qualitative Interviews, quantitative postalische Befragung) zu unterschiedlichen Erhebungszeitpunkten (vor Beginn der Projektmaßnahmen, nach den Seminaren/der Betriebsberatung, drei Monate nach Abschluss der Beratung) sowie durch die standardisierte dermatologische Befunderhebung zu Beginn, zum Ende sowie zu jedem Seminartermin evaluiert worden.

\section{Ergebnisse}

Anhand der Auswertung von 215 Interviews konnten verschiedene Einstellungsmuster herausgearbeitet werden. So stimmten z. B. $46 \%(n=99)$ der Interviewten der Aussage zu, dass gering gerötete oder rauhe Hände „ganz normal“ seien für den Friseurberuf. Damit kann bei dieser Berufsgruppe von einer allgemeinen Akzeptanz geringfügiger Hautveränderungen als obligatorisches Berufsmerkmal gesprochen werden. Deutlich geworden ist auch, dass von den Betroffenen häufig kein ursächlicher Zusammenhang zwischen Erkrankung und der beruflichen Hautbelastung hergestellt wird. So wird die Erkrankung z.B. als „Schicksalsschlag“, „psychisch bedingt“ oder allein durch eine „familiäre Belastung" erklärt.

In den Hautschutzseminaren ging es zum einen um die Vermittlung von Basisinformationen zu den Themen „Hauterkrankungen“ und „Schutzmaßnahmen“. Zum anderen sollen durch praktische Übungen hautschonende Arbeitsweisen trainiert und häufig vorhandene Abwehrhaltungen abgebaut werden. Weiterhin hervorzuheben ist die Bedeutung des Erfahrungsaustausches zwischen den Teilnehmern. In diesem Zusammenhang hat das Seminar teilweise die Funktion einer Selbsthilfegruppe unter professioneller Leitung. Oft konnten jedoch erst durch die Betriebsberatungen die im Seminar erarbeiteten Veränderungsschritte auf den Alltag übertragen werden. Im Rahmen des Projektes sind von uns 103 Beratungen durchgeführt worden, an denen insgesamt 652 Personen teilgenommen haben [3].

Fazit der Projektergebnisse ist, dass von 150 Teilnehmern, die alle Projektmaßnahmen durchlaufen haben, 121 (81\%) das Projekt erfolgreich abgeschlossen haben, d.h., dass die Hautverän-

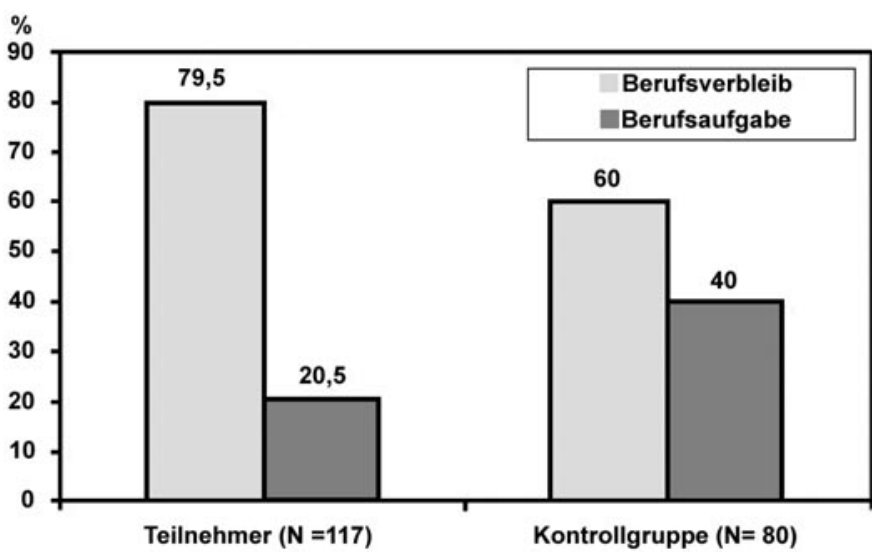

Abb. 2 Sekundäre Individualprävention im Friseurhandwerk: Berufsverbleib, Befragungsergebnisse 3 Monate nach individuellem Projektabschluss.

derungen trotz weiterer Berufstätigkeit abgeheilt waren. Auch ein Vergleich der Teilnehmergruppe mit einer ausschließlich ärztlich behandelten Kontrollgruppe $(n=87)$ hat den Erfolg der gesundheitspädagogischen Maßnahmen unterstrichen. Eine Nachbefragung der Projektteilnehmer frühestens drei Monate nach Abschluss der Maßnahme ergab einen Prozentsatz von $79,5 \%$ derer, die im Beruf verbleiben können im Vergleich zu $60 \%$ in der Kontrollgruppe (Abb. 2).

\section{Tertiäre Prävention}

Von 1994 bis 1999 wurde das Projekt „Stationäre Präventionsmaßnahme für hautkranke Versicherte“ an der Universität Osnabrück durchgeführt. Ziel der auch aktuell noch bestehenden Maßnahme ist es, Patienten mit schweren, ambulant therapieresistenten, beruflich bedingten Hauterkrankungen den Verbleib in ihrem Beruf zu ermöglichen [13].

\section{Konzeption und Methoden}

Die Konzeption der Maßnahmen beinhaltet eine zwei- bis dreiwöchige stationäre Aufnahme zur Behandlung berufsbedingter Hauterkrankungen für solche Versicherte, die schwere und ambulant therapieresistente Dermatosen haben und bei denen ein Zwang zur Berufsaufgabe im Einzelfall droht. Die stationäre Maßnahme wird ergänzt durch eine zwei- bis dreiwöchige Nachbetreuungsphase durch die niedergelassenen Dermatologen am Wohnort der Versicherten unter Fortsetzung der Arbeitskarenz. Diese zeitliche Verlängerung bis zur erneuten vollen beruflichen Hautbelastung basiert auf der Erkenntnis, dass die geschädigte Epidermis zu ihrer vollen Restitution auch nach klinischer Abheilung der Hautveränderungen einen längeren Zeitraum benötigt $[14,15]$.

Durch für die Berufsdermatologie besonders qualifizierte Fachärzte erfolgt eine u. a. allergologische und hautphysiologische Diagnostik, die stadiengerechte Therapie und die Entwicklung eines individuellen Hautschutzprogramms. Diese Maßnahmen werden ergänzt durch gesundheitspädagogische Interventionen in dreierlei Hinsicht: allgemeine Aufklärung und Information über die Entstehung von Berufsdermatosen, spezielle Einzelbe- 
ratungen und Kleingruppenseminare bezüglich geeigneter Hautschutzmaßnahmen sowie individuelles Training hautschonenden Arbeitens unter Anwendung optimierter Schutzmaßnahmen. Um das Ziel Berufsverbleib zu erreichen, werden darüber hinaus allgemeine gesundheitsfördernde Aktivitäten angeboten. Beteiligte Professionen sind heute die Dermatologie, die Ergotherapie, die Kosmetik, die Pädagogik sowie Psychologie und Arbeitsmedizin. Die Projektkonzeption ist orientiert an den RehaRichtlinien des Verbandes deutscher Rentenversicherungsträger [16]. In diesen Richtlinien ist der interdisziplinäre Ansatz für medizinische Rehabilitationsmaßnahmen begründet. So sollen rein medizinisch-körperorientierte Angebote und Leistungen zurücktreten gegenüber verhaltensorientierten Maßnahmen in Gruppen und verschiedene Therapiemaßnahmen in ein gemeinsames Klinikkonzept integriert werden.

Die Versicherten, die nachbehandelnden Hautärzte wie auch die zuständigen Berufsgenossenschaften werden ein Jahr nach dem stationären Aufenthalt schriftlich befragt, um die Effizienz dieser Maßnahme im Sinne der Ergebnisqualität beurteilen zu können.

\section{Ergebnisse}

Die bisherige Auswertung umfasst die Jahre 1994 bis 1999. Von 490 an die Patienten versandten Fragebogen kamen 352 zurück (Rücklauf 71,8\%).

Abb. 3 zeigt, dass ein Berufsverbleib bei 65,9\% $(n=232)$ der Versicherten möglich war. Wenn eine Berufsaufgabe erfolgte, war dies zu $23 \%(n=81)$ mit der berufsbedingten Hauterkrankung begründet. Eine Berufsaufgabe aus anderen Gründen erfolgte zu $8,5 \%$, $(\mathrm{n}=30)$, keine Angabe zum Grund der Berufsaufgabe machten $1,1 \%$ der Befragten $(n=4)$. Auf die Frage, ob die empfohlenen Schutzmaßnahmen regelmäßig angewendet werden, antworteten 81,3\% $(n=286)$ der Befragten mit ,ja“ und 6,8\% $(n=24)$ mit „nein“, keine Angabe machten 9,9\% ( $\mathrm{n}=35)$, sonstige Angaben erfolgten von $11,9 \%(n=42)$ der Befragten. Dass der Arbeitgeber Schutzhandschuhe zur Verfügung stellt, bejahten $55,7 \%$ $(\mathrm{n}=196)$ der Befragten, mit „nein“ antworteten 18,2\% $(\mathrm{n}=64)$ und keine bzw. sonstige Angaben machten 9,9\% $(n=35)$ bzw. $16,2 \%(n=57)$.
Diskussion

Als Basis und Legitimation für Interventionsstudien zur Prävention berufsbedingter Hauterkrankungen sollten epidemiologische Studien vorliegen. Die hier dargestellte Studie von Uter et al. [20] hat u.a. den potenziellen Nutzen von Hautschutzmaßnahmen im Sinne einer „Bevölkerungsstrategie“ (z. B. alle Friseure) im Vergleich zu einem denkbaren „Hochrisikoansatz“ (z.B. nur Friseure mit atopischer Hautdisposition) belegt. Somit kann die Konzeption von Interventionsstrategien für alle Beschäftigten im Friseurhandwerk begründet werden. Durch die von Schlesinger et al. [9] vorgelegten Ergebnisse der Langzeitstudie bei Friseurauszubildenden können inhaltliche Schwerpunkte abzuleitender Präventionsmaßnahmen identifiziert werden. So zeigt die von 1994 auf 1999 gestiegene Diskrepanz zwischen aufgetretenen Hautveränderungen und der Konsultation eines Dermatologen beispielsweise, dass die Auszubildenden möglichst frühzeitig, bereits bei geringfügigen Hautveränderungen, durch die Lehrer bzw. durch im Unterricht vermittelte Information und Motivation zu einem Hautarztbesuch zu bewegen sind. Die von Schlesinger et al. und Uter et al. [8,9] dargestellten Ergebnisse und abgeleiteten Forderungen nach Interventionen decken sich mit anderen europäischen Studien $[17,18]$.

Die kontrollierten Studien zur primären [12] und sekundären [3] Prävention von Hauterkrankungen im Friseurhandwerk haben gezeigt, dass gesundheitspädagogische Interventionen in Ergänzung zu einer berufsdermatologischen Betreuung im Vergleich zur ambulanten dermatologischen Therapie erfolgreich sind. Weitere Studien zur primären Prävention von Hauterkrankungen bei Auszubildenden in hautbelastenden Berufen wie dem Nahrungsmittel- und Backgewerbe sowie der Krankenpflege bestätigen diese Ergebnisse [19,20]. Erste Ergebnisse zur sekundären Individualprävention von Hauterkrankungen in der Altenpflege sprechen für die Übertragbarkeit des erstmals an hauterkrankten Friseuren erprobten Konzeptes [21].

Einer von Dusseldorp et al. [22] durchgeführten Metaanalyse zur Effektivität von Patientenschulungen bei koronaren Herzerkrankungen zufolge waren solche Ansätze am erfolgreichsten, die ge-

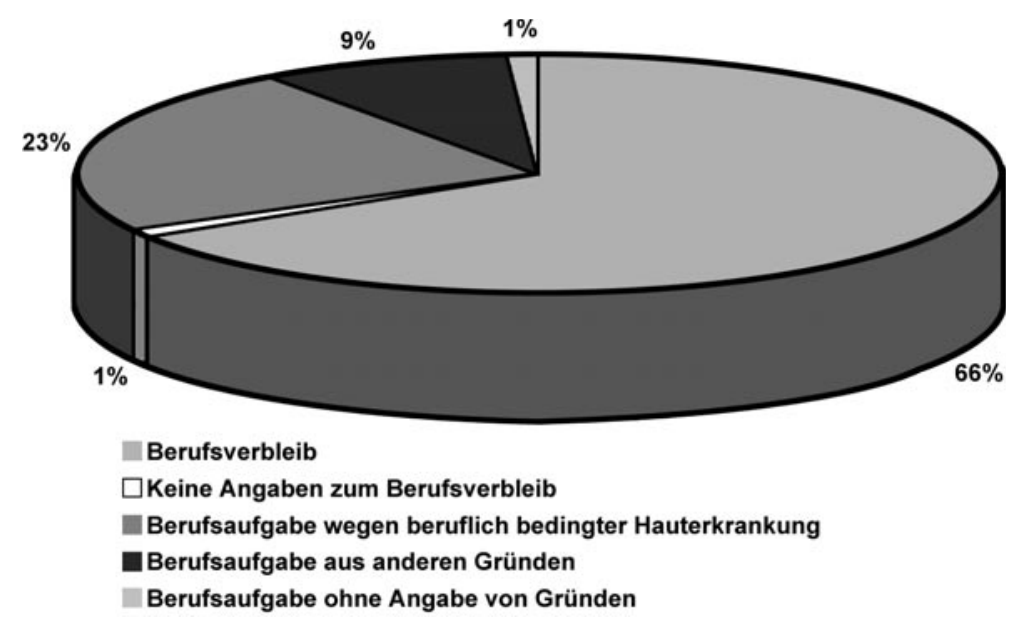

Abb. 3 Tertiäre Prävention/stationäres Heilverfahren, Patientenbefragung 1994 - 1999: Berufsverbleib 1 Jahr nach stationärer Aufnahme, $\mathrm{n}=352$. 
sundheitserzieherische und gesundheitspsychologische (vor allem Stressmanagement-)Ansätze integriert haben, wie dies beispielsweise bei der dargestellten stationären Maßnahme für Personen mit ambulant therapieresistenten, berufsbedingten Hauterkrankungen erfolgt ist [13]. Die in den letzten zehn Jahren bundesweit feststellbaren Erfolge präventiver Maßnahmen bezüglich der Hauterkrankungen im Friseurhandwerk [5] können zum Teil erklärt werden mit der Verbesserung von administrativen Regelwerken zum Arbeitsschutz [23] sowie mit der durch den zuständigen Unfallversicherungsträger erfolgten Einführung des beschriebenen Präventionsprogramms zur sekundären Individualprävention im Friseurhandwerk in die Regelversorgung hauterkrankter Friseure und mit den verbesserten stationären Heilverfahren [24]. Diese Entwicklungen sprechen für eine weitere Implementierung gesundheitspädagogischer Maßnahmen in die Prävention von Berufsdermatosen. Diese sollten wissenschaftlich begründet sein und entsprechend evidenzbasierter Qualitätsstandards durchgeführt und evaluiert werden, um fundierte Aussagen über die (Langzeit-)Effektivität solcher Programme zu ermöglichen.

Zusammenfassend können die von uns konzipierten gesundheitspädagogischen Maßnahmen immer dann sinnvoll eingesetzt werden, wenn Krankheitsentstehung und Krankheitsverlauf stark von dem Verhalten bzw. der Motivation des Einzelnen und den durch das soziale bzw. berufliche Umfeld geprägten Verhältnissen abhängig sind. Resultate einer praktisch erfolgreichen Gesundheitspädagogik sind a) ein verändertes Gesundheitsbewusstsein, b) die Handlungskompetenz bezüglich Gesundheitsgefährdungen oder Krankheitssymptomen und c) die Konfliktfähigkeit, um tradierte gesundheitsgefährdende Verhaltensmuster zu verändern.

\section{Literatur}

${ }^{1}$ Hornstein OP, Haneke E, Ummenhofer B. Klinik-integrierte Rehabilitationstherapie chronischer Ekzemkrankheiten. Ärztliche Erfahrungen mit einem neuen Rehabilitationsmodell. Stuttgart: Thieme, 1981

${ }^{2}$ Wulfhorst B. Theorie der Gesundheitspädagogik. Reihe Grundlagentexte Gesundheitswissenschaft München, Weinheim: Juventa, 2002

${ }^{3}$ Wulfhorst B. Sekundäre Prävention von Hauterkrankungen im Friseurhandwerk. Reihe „Studien zur Prävention in Allergologie, Berufsund Umweltdermatologie“. Osnabrück: Universitätsverlag Rasch, 2001

${ }^{4}$ Wigger-Alberti W, Elsner P. Fluoreszenz im Wood-Licht - Aktueller Einsatz in der dermatologischen Diagnostik, Therapiekontrolle und Prävention. Hautarzt 1997; 48: $523-527$

${ }^{5}$ Dickel H, Kuss O, Schmidt A, Diepgen TL. Impact of preventive strategies on trend of occupational skin disease in hairdressers: population based register study. BMJ 2002; 324: 1422-1423
${ }^{6}$ Schwanitz HJ. Prävention chronischer Friseurekzeme. Allergologie 1993; $16: 408-412$

${ }^{7}$ Schwanitz HJ, Uter W. Interdigital dermatitis: sentinel skin damage in hairdressers. British Journal of Dermatology 2000; 142: 1011 - 1012

${ }^{8}$ Uter W, Pfahlberg A, Gefeller O, Schwanitz HJ. Hand dermatitits in a prospectively followed cohort of hairdressing apprentices. Final results of the POSH-study. Contact Dermatitis 1999; 41: 280-286

${ }^{9}$ Schlesinger T, Revermann K, Schwanitz HJ. Dermatosen bei Auszubildenden des Friseurhandwerks in Niedersachsen. Ein Vergleich zwischen 1989, 1994 und 1999. Dermatol. Beruf Umwelt 2001; 49: 185 192

${ }^{10}$ TRGS Technische Regeln für Gefahrstoffe 530 - Friseurhandwerk. Bundesarbeitsblatt 1992; 9: 41 - 45

${ }^{11}$ Budde-Wamhoff U. Unterrichtsmappe Gesundheitsförderung im Friseurhandwerk. Informationen und Materialien zum Unterricht. Hautschäden und Hautschutz. Berlin: Cornelsen, 1991

${ }^{12}$ Riehl U. Interventionsstudie zur Prävention von Hauterkrankungen bei Auszubildenden des Friseurhandwerks. Osnabrück: Universitätsverlag Rasch, 2001

${ }^{13}$ Schwanitz HJ. Tertiäre Prävention von Berufsdermatosen. Bericht über das Forschungsprojekt „Stationäre Präventionsmaßnahme für hautkranke Versicherte“. Dermatol. Beruf Umwelt 2002; 50: 212 - 217

${ }^{14}$ Choi JM, Lee JY, Cho BK. Chronic irritant contact dermatitis: recovery time in man. Contact Dermatitis 2000; 42: 264-269

${ }^{15}$ Fartasch M. Human Barrier Formation and Reaction to Irritation. In: Elsner P, Maibach HI (ed). Irritant Dermatitis. New Clinical and Experimental Aspects. Curr Probl Dermatol. Basel: Karger, 1995: 95 - 103

${ }^{16}$ Verband deutscher Rentenversicherungsträger VDR (Hrsg). RehaKommission Abschlußberichte. Bd. VI Arbeitsbereich Wissenschaft und Lehre. Frankfurt: 1991

${ }^{17}$ Ling TC, Coulson ICH. What do trainee hairdressers know about hand dermatitis? Contact Dermatitis 2002; 47: 227-231

${ }^{18}$ van der Walle HB. Dermatitis in hairdressers. (II) Management and prevention. Contact Dermatitis 1994; 30: 265-270

19 Bauer A, Kelterer D, Stadeler M, Schneider W, Kleesz P, Wollina U, Elsner $P$. The prevention of occupational hand dermatitis in bakers, confectioners and employees in the catering trades: preliminary results of a prevention program. Contact Derm 2001; 44: 85-88

${ }^{20}$ Held E, Wolff C, Gyntelberg F, Agner T. Prevention of work-related skin problems in student auxiliary nurses: an intervention study. Contact Dermatitis 2001; 44: 297-303

${ }^{21}$ Klippel U, Schürer N, Wulfhorst B, Schwanitz HJ. Berufsdermatosen in der Altenpflege: Früherkennung und -intervention sind Ziel der sekundären Individualprävention. Pflegezeitschrift 2001; 8: 575-578

22 Dusseldorp E, von Elderen T, Maes S, Meulmann J, Kraaij VA. Metaanalysis of psychoeducational programs for coronary heart disease patients. Health Psychology 1999; 18: 506-519

${ }^{23}$ Schröder C, Wulfhorst B, Schwanitz HJ. Welche Relevanz haben Technische Regeln Gefahrstoffe (TRGS) auf die Arbeitsdermatologie? Allergologie 1998; 21: 393

${ }^{24}$ Rojahn K, Brandenburg S, Remé T. Berufliche Wiedereingliederung von Beschäftigten bei Haut- und Atemwegsbeschwerden (BK 5101/ BK 4301/ 4302). ErgoMed 2001; 6: $193-198$

${ }^{25}$ Bauer A, Kelterer D, Bartsch R, Pearson J, Stadeler M, Kleesz P, Elsner P, Williams H. Skin protection in bakers' apprentices. Contact Dermatitis 2002; 46: $81-85$ 